\title{
Artificial Intelligence Based Sclera Vein Pattern Recognition for Human Identification
}

\author{
Onkar G Hastak \\ PG Scholar, Electronics Engineering \\ Bapurao Deshmukh College of Engineering,Sevagram, Maharashtra, India \\ Prof.V.R.Ingle \\ Professor, Electronics Engineering, \\ Bapurao Deshmukh College of Engineering,Sevagram, Maharashtra, India \\ Prof.D.R.Dandekar \\ Professor, Electronics Engineering, \\ Bapurao Deshmukh College of Engineering,Sevagram, Maharashtra, India
}

\begin{abstract}
In this paper, we propose Artificial Intelligence based sclera vein pattern recognition for human identification. Here sclera segmentation is performed by Fuzzy clustering Means (FCM). Since the sclera vein patterns are not clearly visible, image enhancement is required. A Fuzzy logic-based Brightness Preserving Dynamic Fuzzy Histogram Equalization (BPDHE) is used to enhance the sclera vein patterns. Dense Local Binary Pattern (D-LBP) is used for feature extraction. Finally, for matching purposes Artificial Neural Network (ANN) with back propagation is used. The UBIRIS.v1 database is used here for experimentation. The experimental results achieve matching overall accuracy of $\mathbf{9 8 . 8 8} \%$ and $\mathbf{8 0 . 3 1 \%}$ average noise removed from noisy images to perform matching.
\end{abstract}

Keywords - Biometrics, Sclera vessels Patterns, FCM, BPDHE, D-LBP, ANN, UBIRIS.

\section{INTRODUCTION}

Human identification is a very essential and useful method in this populated world. The term 'identification' means the act or process of "establishing the identity of, [or] recognizing", or "the treating of a thing as identical with another". The human identification has become very essential in several fields of our daily life like banking sector, defense sector, industrial sector, educational sector, medical sector etc. The human identification process gives us a more secure way to authenticate a human being.

There are different ways are present to identify a human being. Those are:

Names - Different names are given to different human beings for identification. This is a complete social process for identification. But sometimes the names become non-unique for more than one human.

Codes - Sometimes the organizations assigns unique code for their employees for identification. This can be done in manual or automated way. This process became very useful for organization purpose.

Knowledge based identification - People can be recognized by combination of social data. Examples of data used for this purpose are one's family and given names, prior names, father's name, mother's name, mother's and grandmothers' maiden names, date and place of birth, address, marital status, religion, and occupation.

Token-Based Identification - A 'token' is some 'thing' which a person has in his or her possession, in particular documentary evidence. Commonly used documents are birth and marriage certificate, passport, driver's license, employer-issued building security card, credit card, club membership card, statutory declaration, affidavit, or letter of introduction.

Biometrics - Every human being has many different features in the human body which can be used to identify a human being. In medical field there are several test technique to evaluate distinct features of human body like blood group, bone structure, DNA structure etc. Biometrics is the human identification using different physiological, biological, or behavioral characteristics, traits, or habits.

Many biometric systems are in used. Though biometric identification based on digital fingerprints, retinal scans, 
facial characteristics, gesture, and voice patterns are distinctive to each and every individual and are considerably more reliable than the traditional token-based or knowledge-based systems in differentiating between an authorized and a non-authorize person. Though, till now no biometric system claims the properties of a perfect authentication system.

Among various biometrics, biometrics including iris and retina are known as the most accurate biometrics. But a few disadvantages such as the capture of iris images requires the cooperation of the user since an off-axis iris image can deteriorate system performance, and retina scanning requires contact with an eye-piece which is far from being user friendly. Apart from iris and retinas, the human eye has an ocular surface known as the sclera. To date, this biometric has not been thoroughly studied and little is known about its utility.

To our knowledge, the first recognized work on sclera biometrics is recorded in [1]. Automatic segmentation processes of sclera is proposed in [4], [6] and many features such as LBP [9], GMCL [8] are used for recognition. Work on multi-angled sclera recognition $[2,7]$ as well as multimodal eye recognition techniques $[3,5,10]$ are also proposed using sclera and the iris.

This present work proposes a whole biometric system for personal identification based on sclera vessels. Here sclera segmentation was performed by Fuzzy C-means clustering. A new preprocessing approach for vein highlighting is proposed here by the Brightness Preserving Dynamic Histogram Equalization (BPDHE). Dense Local Binary Pattern (D -LBP) is used for sclera feature extraction also new in the literature.

The paper is organized in a pattern as follows. Section II explains the proposed approach: segmentation, preprocessing of the sclera images i.e. sclera vessel enhancement process, feature extraction and . In Section III, describes some results the experimental results and in Concluding remarks are given in Section IV.

\section{PROPOSED APPROACH}

In this section, the proposed system sclera recognition process involves: segmentation of sclera (FCM), a sclera vein pattern enhancement technique (BPDHE) and sclera feature extraction((D-LBP) is explained. The proposed system block diagram in shown in figure 1.

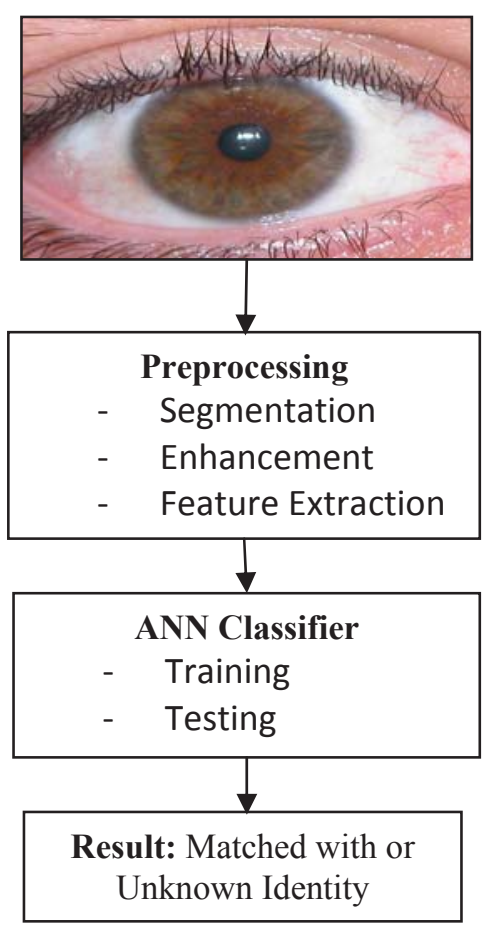

Figure 1: Proposed System Block Diagram

\subsection{SCLERA SEGMENTATION}

The sclera is a white portion of connective tissue and blood vessels surrounding the iris. This portion of blood vessels inside the sclera portion is randomly oriented which creates a pattern which is used for biometric 
identification. Segmentation is the first step for most biometric related research. Similarly in sclera biometrics, accurate segmentation

is very important, otherwise, an incorrect segmentation can reduce the pattern available, but also it can introduce other patterns such as eyelashes and eyelids. Here sclera segmentation is performed by a Fuzzy C-means clusteringbased segmentation proposed in [20]. Fuzzy C-means is a method of clustering which allows one piece of data to belong to two or more clusters $[18,19]$.

A experimentation of the proposed algorithm is carried out on sclera images from different modalities. The results confirm its effectiveness for sclera image segmentation. The number of clusters considered here was three and index three. The segmentation was performed on grey images. Figure 2(c) shows the Fuzzy C means-based sclera segmentation of 1(a) index 1. Figure 2(d) shows the Fuzzy C means-based sclera segmentation of 2(a) index 2 and Figure 2(f) shows the Fuzzy C means-based sclera segmentation of 2(a) index 3 and 2(b) grey image of 2(a).

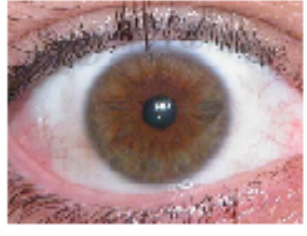

(a)

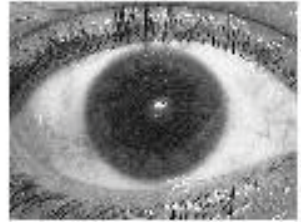

(b)

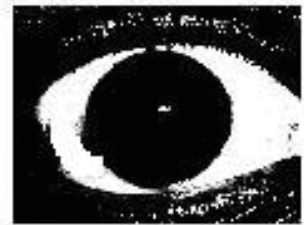

(c)

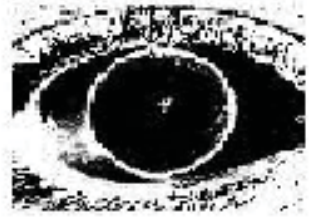

(d)

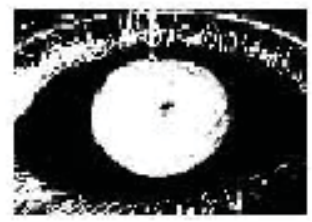

(e)

Figure 2: (a)original image, (b) grey image of (a). Figure (c) shows the Fuzzy C means-based sclera segmentation of 1(a) index 1.Figure (d) shows the Fuzzy C means-based sclera segmentation of (a) index 2 and Figure (e) shows the Fuzzy C means-based sclera segmentation of (a) index 3.

Here the Fuzzy C means-based sclera segmentation of index 1 is used as mask for further experimentation.

\subsection{SCLERA VESSEL STRUCTURE ENHANCEMENT}

The vessels in the sclera are not clearly visible, so in order to make them clearly visible, image enhancement is required. Brightness Preserving Dynamic Fuzzy Histogram Equalization is on the green channel of the sclera image as the sclera vessel patterns are most prominent in the green channel as shown in Figure 3(c)) to make the vessel structure more clear as shown in Figure 4.

Brightness preserving dynamic histogram equalization technique improves its brightness preserving and contrast enhancement ability while reducing its computational complexity.

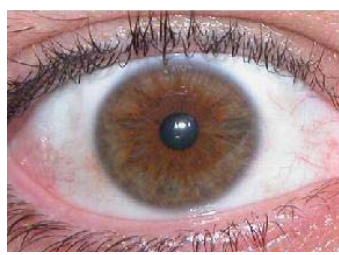

(a)

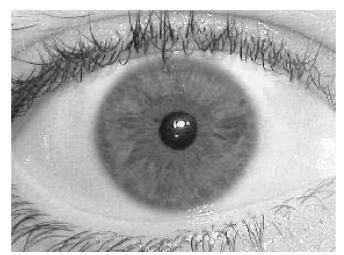

(b)

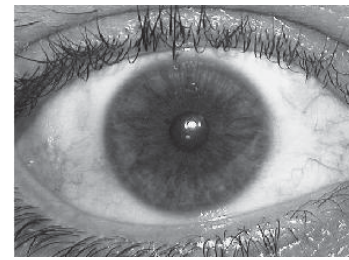

(c)

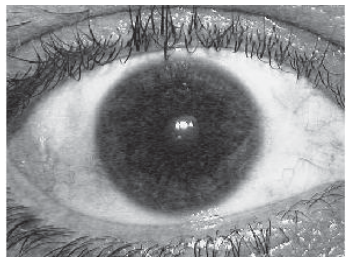

(d)

Figure3. (a) The original RGB image, (b) The red channel component of (a),(c) The green channel component of (a), and (d) blue channel component of (a),

\subsection{FEATURE EXTRACTION METHOD}

In past years, a certain level of good performance has been achieved by several researchers. Although there exists security weakness in their extracted templates. Inherently, the extracted biometric template cannot be used as a passwords and personal identification number(PIN) if compromised. This can be significant issue particularly when many application share a biometric and the biometric template. Since Dense Local binary pattern(D-LBP) algorithm is used for feature extraction figure 1 shows extracted sclera vessel structure. D-LBP based approach is expected to be easily extended to multimodal sclera recognition. and also for Artificial Intelligence Based Human Identification. 
Figure 4: Extracted Sclera Vessel structure

\subsection{RECOGNITION/MATCHING}

Once we obtain sclera vein features, the next step is vein pattern recognition/matching. In this section, we introduce Neural Network. We give a brief description of the Neural Network method.

\section{Neural Networks}

Artificial neural networks are composed of interconnecting artificial neurons. Artificial neural networks is used for solving artificial intelligence problem without necessarily creating a model of a real biological system. Neural network give effective results for solving multiple class classification problems. Figure shows basic layout of the neural networks.

Neural network has three types of layers: input layer, output layer and hidden layer. Hidden layer does intermediate computation before directing the input to output layer. Back propagation can also be considered as ageneralization of delta rule. When back propagation network is cycled, and input pattern is propagated forward to the output units through the intervening input to hidden and hidden to output weights. Neural network have been widely used in image and signal processing.

Recognition/ Matching using ANN required two steps: Training (Learning) and Testing.

\section{EXPERIMENTAL RESULTS}

The experimental setup and the results of our proposed work are explained in this section.

\subsection{DATA SET}

In order to experiment the performance of the proposed method, the UBIRIS.v1 database [13] was utilized for our experiments. This database consists of 1877 RGB images from 241 persons. These images are minimize with noise factors, those relative to reflection, luminosity and contrast.

\subsection{RESULTS OF SEGMENTATION}

The results of segmentation are discussed here.

\section{2(A) SEGMENTATION RESULTS}

In these experiments, different quality images were used. Some of them were not occluded having good quality of sclera regions visible, some of them are of medium quality and the third type was of poor quality with respect to sclera region visibility, some closed eye images were also used. In the experiments, some sample images is used for segmentation. Examples of FCM segmentation with class size $=3$ are given below in Figure 6.

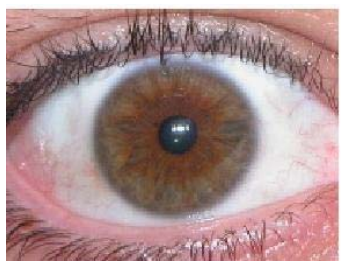

(a)

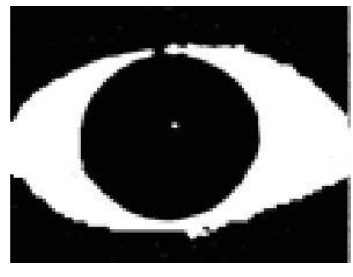

(b)

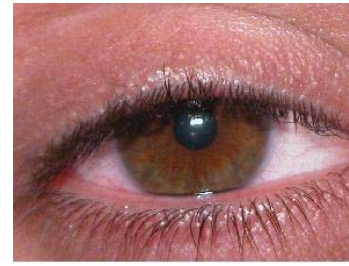

(c)

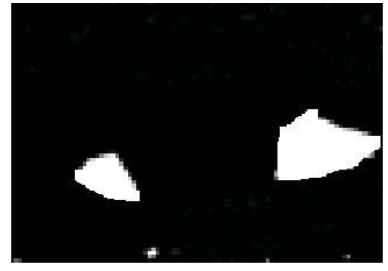

(d)

Figure 6: Examples segmented images. (a) \& (c) are the original images, (b) \& (d) are segmented images

3.2(B) RESULTS FOR SCLERA VESSEL ENHANCEMENT

Experimental results of the enhancement techniques BPDHE used for sclera vessel enhancement is shown in figure

\subsection{FEATURE SELECTION}


For feature extraction, few local features extraction techniques such as Dense SIFT (Scale Invariant Feature Transform), Dense LBP (Local Binary Pattern) and dense color can be used. The previous results of researchers show that dense LBP produces the best results. Hence, dense LBP was used for feature extraction. Figure 4 shows extracted feature sclera vein pattern used to train ANN.

\subsection{MATCHING}

ANN based matching technique is used in experimentation and while testing salt and pepper noise is added in image which is then removed using filtering. After this matching is performed the results of which is summarized below:

\begin{tabular}{|c|c|c|c|c|c|}
\hline \multirow{2}{*}{ Training(T) } & \multicolumn{2}{|l|}{ Testing } & \multicolumn{2}{l|}{ Matching Accuracy(\%) } & Overall \\
\cline { 2 - 5 } & \multicolumn{1}{|l|}{ Train } & Untrain & Ttrain & Untrain & Accuracy(\%) \\
\hline 20 & 20 & 20 & 100 & 100 & 98.88 \\
\hline 30 & 30 & 20 & 96.66 & 100 & \\
\hline 30 & 30 & 30 & 96.66 & 100 & \\
\hline
\end{tabular}

\section{CONCLUSIONS}

In this paper we proposed a method of artificial intelligence based sclera vein pattern recognition for human identification. For segmentation, a Fuzzy C-means-based segmentation approach is proposed. Fuzzy logic-based Brightness Preserving Dynamic Fuzzy Histogram Equalization(BPDFHE) is used for sclera enhancement. Dense Local Binary Pattern(D-LBP) is used for feature extraction. The proposed approach is experimented on the UBIRIS.v1database. Experimented images gives substantially good sclera vein pattern which is used for artificial intelligence based human identification. The experimental results achieve matching overall accuracy of $98.88 \%$ and $80.31 \%$ average noise removed from noisy images to perform matching.

\section{REFERENCES}

[1] R. Derakhshani, A. Ross, and S. Crihalmeanu. A new biometric modality based on conjunctival vasculature. Proceedings of Artificial Neural Networks in Engineering: pp.1-8, 2006.

[2] Z. Zhou, Y. Du, N. L. Thomas, and E. J. Delp. Multi angled sclera recognition. IEEE Workshop on Computational Intelligence in Biometrics and Identity Management: pp.103-108, 2011.

[3] Z. Zhou, Y. Du, N. L. Thomas, and E. J. Delp. Multimodal eye recognition. Proceedings of the International Society for Optical Engineering, 7708(770806): pp.1-10, 2010.

[4] Z. Zhou, Y. Du, N. L. Thomas, and E. J. Delp. A new biometric sclera recognition. IEEE transaction on System, Man And Cybernatics PART A: System And Human, 42(3): pp.571-583, 2012.

[5] Z. Zhou, Y. Du, N. L. Thomas, and E. J. Delp, Quality Fusion Based Multimodal Eye Recognition, IEEE International Conference on Systems, Man, and Cybernetics: pp.1297-1302, 2012.

[6] M. H. Khosravi and R. Safabakhsh, Human eye sclera detection and tracking using a modified time-adaptive self-organizing map, Pattern Recognition, 41: pp.2571 - 2593, 2008.

[7] S. Crihalmeanu and A. Ross, Multispectral sclera patterns for ocular biometric recognition, Pattern Recognition Letters, 33: pp.1860-1869, 2012.

[8] S. P. Tankasala, P. Doynov, R. R. Derakhshani, A. Ross and S. Crihalmeanu, Biometric Recognition of Conjunctival Vasculature using GLCM Features, International Conference on Image Information Processing : pp.1-6, 2011.

[9] K. Oh and K. Toh, Extracting Sclera Features for Cancelable Identity Verification, 5th IAPR International Conference on Biometric: pp.245-250, 2012.

[10] V. Gottemukkula, S. K. Saripalle, S. P. Tankasala, R. Derakhshani, R. Pasula and A. Ross, Fusing Iris and Conjunctival Vasculature: Ocular Biometrics in the Visible Spectrum, IEEE Conference on Technologies for Homeland Security: pp.150-155, 2012.

[11] T. Mäenpää, M. Pietikäinen, Texture Analysis with local binary Patterns $\backslash$, in C.H. Chen, P.S.P. Wang (eds.): Handbook of Pattern Recognition and Computer Vision, $3^{\text {rd }}$ edn. World Scientific, pp. 197-216, 2005.

[12] S. Lazebnik, C. Schmid, and J. Ponce, "Beyond bags of features: Spatial pyramid matching for recognizing natural scene categories," In Proc. Computer Vision and Pattern Recognition CVPR, 2, pp. 2169-2178, 2006.

[13] Hugo Proença and, Luís A.Alexandre, UBIRIS: A noisy iris image database, Proceed. of ICIAP 2005 - Intern. Confer. on Image Analysis and Processing, 1, pp.970-977, 2005.

[14] A. Das, U. Pal, M. F. A. Ballester and, M. Blumenstein, A New Method for Sclera Vessel Recognition using OLBP, In Proc. Chinese Conference on Biometric Recognition ,LNCS 8232, pp. 370-377, 2013.

[15] M. A. F. Ballester, A. Morales, A. Das, M. Blumenstein and U. Pal, Model based Sclera vessels segmentation with SIFT Recognition and 
its combination with Iris", appeared in Spanish biometric consodium, VII Jornadas de Reconocimiento Biometrico de Personas, pp. 68-76, 2013

[16] A. Das, U. Pal, M. F. A. Ballester and, M. Blumenstein, Sclera Recognition Using D-SIFT, In Proc. 13th International Conference on Intelligent Systems Design and Applications, pp. 74-79, 2013.

[17] A. Das, U. Pal, M. Blumenstein and M. F. A. Ballester, Sclera Recognition - A Survey, In Proc. Recent Advancement in Computer Vision and Pattern Recognition, pp. 917-921, 2013.

[18] J. C. Dunn, A Fuzzy Relative of the ISODATA Process and Its Use in Detecting Compact Well-Separated Clusters, Journal of Cybernetics 3: pp. 32-57, 1973.

[19] J. C. Bezdek, Pattern Recognition with Fuzzy Objective Function Algoritms, Plenum Press, New York, 1981.

[20] Abhijit Das, Umpada Pal, Miguel Angel Ferrer Ballester and Michael Blumenstein. Fuzzy Logic Based Sclera Recognition. IEEE International Conference on Fuzzy System, pp. 561-568, Jul. 6-11,2014.

[21] D. Sheet, H. Garud, A. Suveer, J. Chatterjee and M. Mahadevappa ,Brightness Preserving Dynamic Fuzzy Histogram international Equalization, IEEE Trans., Consumer Electronics, 6(4), pp. 2475 - 2480, Nov.2010. 